\title{
Group Delay measurements of ultra-broadband pulses generated in highly nonlinear fibers
}

\author{
Jan Szczepanek, ${ }^{1}$ Tomasz M. Kardaś, ${ }^{2}$ and Yuriy Stepanenko ${ }^{2, *}$ \\ ${ }^{1}$ Faculty of Physics, University of Warsaw, Pasteura 5, 02-093 Warsaw, Poland \\ ${ }^{2}$ Institute of Physical Chemistry Polish Academy of Sciences, Kasprzaka 44/52, 01-224 Warsaw, Poland
}

Received November 22, 2016; accepted December 19, 2016; published December 31, 2016

\begin{abstract}
Ultra broadband supercontinuum pulses are commonly used as a source of different wavelengths from a wide spectral bandwidth or as a source of very short pulses. However, the processes responsible for wide spectral broadening are still under investigation. In this paper we examine the temporal and spectral characteristics of pulses broadened upon propagation in highly nonlinear photonics crystal fibers with different dispersion profiles. Generated supercontinuum pulses were experimentally characterized using a crosscorrelation frequency resolved optical gating technique.
\end{abstract}

Pulses with an ultra-broadband spectrum are often produced through supercontinuum generation in different media. These include crystals (like sapphire, calcium fluoride, barium borate (BBO) [1]), diamonds [2], liquids [3], gases or other solid materials [4]. Here, the supercontinuum generation is caused by critical selffocusing of a laser beam and the following photoionization of the medium [5]. Highly nonlinear processes and a threshold like the behavior of these processes lead to limited stability of these supercontinua [4]. On the other hand, a popular, stable and reliable method, available in most of commercial solutions, relies on supercontinuum generation in allnormal dispersion highly nonlinear optical fibers [6]. A small mode field area together with high peak power of the seeding pulse ensure high pulse intensity necessary for effective broadening of the pulse by nonlinear effects accumulated through a respectively significant medium length. The intensity is, however, kept bellow the photoionization threshold. The supercontinuum generation in fibers is triggered by nonlinear processes, namely, selfphase modulation (SPM) and stimulated Raman scattering (SRS) [4]. A particular supercontinuum generation regime is, however, governed by the linear properties of fibers. Thus, for fibers with a dispersion curve zero crossing point located in the vicinity of the pump wavelength, the interesting dynamic involving soliton fission, dispersive wave, Raman solitons, etc. can be observed as the velocities of different spectral components can be equalized by nonlinear effects [6].

* E-mail: stepanenko@ichf.edu.pl
On the other hand, stable and repeatable supercontinua are generated in all normal dispersion fibers where newly generated spectral components constantly recede from each other, and thus do not have the chance to interact for longer times [7]. Spectral broadening mechanisms have been investigated with the Cross-correlation Frequency Resolved Optical Gating (XFROG) technique [8] by several research groups [9-11]. However, the processes responsible for extreme spectral broadening are still under intensive investigation [12-14].

In this paper we will focus on temporal and spectral dynamics of pulses broadened in two types of Photonics Crystal Fibers (PCF) possessing different dispersion profiles. Supercontinuum pulses were characterized with the XFROG technique. For spectral broadening two commercially available fibers produced by NKT Photonics were used. Namely, $0.31 \mathrm{~m}$ of NL-1050-NEG-1 which is an all normal dispersion fiber for the spectral range $600 \div 1400 \mathrm{~nm}$ and $1.16 \mathrm{~m}$ of LMA-PM-5 fiber with the Zero Dispersion Wavelength (ZDW) around $\sim 1060 \mathrm{~nm}$. Fiber lengths were chosen in such a way that the nonlinear length of both fibers was similar [15]. The $\beta_{2}$ (group velocity dispersion) coefficient curves for both fibers calculated from data provided by NKT Photonics are presented in Fig. 1.

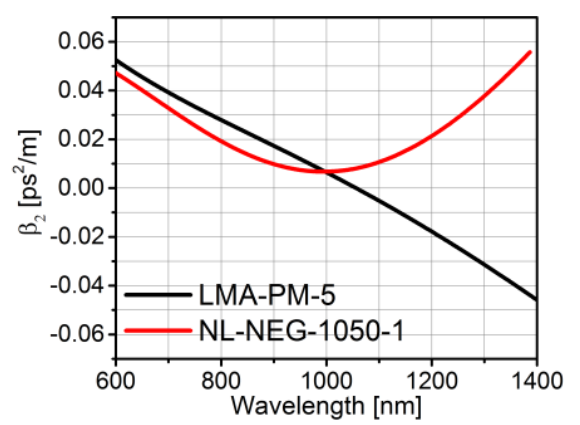

Fig. 1. Group velocity dispersion characteristics of NL-NEG-1050-1 and LMA-PM-5 Photonic Crystal Fibers (PCF).

Nonlinear fibers were pumped by a home-made all-PMfiber system containing an ultrafast all-normal dispersion oscillator and amplifier (setup similar to the one presented earlier in [16]). After the amplification pulses were compressed with a standard grating compressor which 
resulted in pulse duration below 200fs for the whole range of pump powers. The power characteristic of the amplifier is presented in Fig. 2. The energy of the seeding pulse was at a level of $52 \mathrm{~nJ}$ for the maximum pump power. The allPM-fiber setup generated pulses centered around 1030nm at the $12.6 \mathrm{MHz}$ repetition rate. The spectrum and spectral phase of the pulse with the maximum energy are presented in the top-left inset in Fig. 2, the pulse temporal intensity measured with Spectral Phase Interferometry for a Direct Electric-field Reconstruction (SPIDER) [17] apparatus is presented in the bottom-right inset.

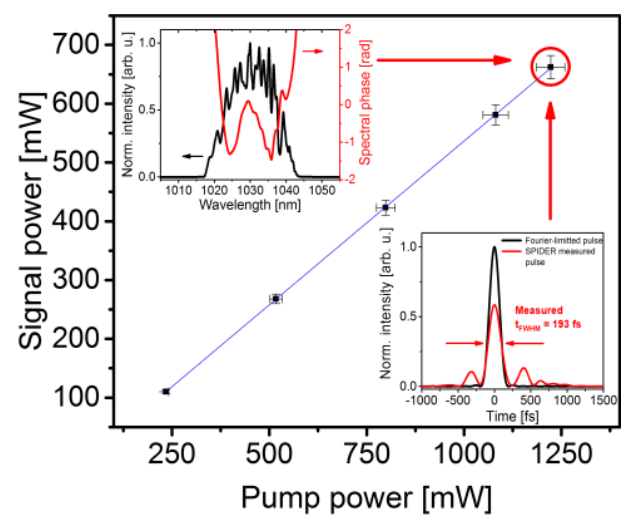

Fig. 2. The seed source characterize with linear change of signal power during increasing the amplifier pump power level.

The seeding pulse was coupled into the NL-1050-NEG-1 fiber by means of the Aspheric Lens (AL; Thorlabs A240B) with the efficiency around $\sim 60 \%$. For the LMA-PM-5 fiber a $10 \times$ microscope objective was used with $\sim 40 \%$ efficiency. The Numerical Aperture (NA) of coupling optics and the laser spot size was matched to the NA and mode field diameter of the fibers, respectively. The broadened pulses were investigated with the home-made XFROG apparatus. The scheme of the setup is presented in Fig. 3. A compressed pulse from the amplifier was divided by means of the $10 / 90$ beam splitter (BS). The $90 \%$ part of the intensity was used for seeding the PCF, the rest of the pulse was sent to the delay line. The first half wave-plate $(\lambda / 2)$ in the setup before the PCF was used to couple the linearly polarized light from the amplifier to the slow axis of the PM fiber. The second $\lambda / 2$ was used with the crystal polarizer to establish the polarization state for the XFROG diagnostic setup and to measure the Polarization Extinction ratio of generated supercontinuum pulses. The broad supercontinuum pulse was mixed with $10 \%$ of the ultrashort pulse from the amplifier in the $20 \mu \mathrm{m}$ thick BBO type I crystal. The spectrum of the pulse produced by the sum frequency process in respect to different delays was registered by means of an Ocean Optics USB4000 spectrometer (span: 177 $\div 895 \mathrm{~nm}$, resolution: $0.22 \mathrm{~nm}$ ).

Two exemplary maps of group delay distribution measurements for the highest energies of the pulse are

shown in Fig. 4 (a) and (b). In Fig. 4 c) and d) the changes of the group delay distribution for different pump power levels are presented.

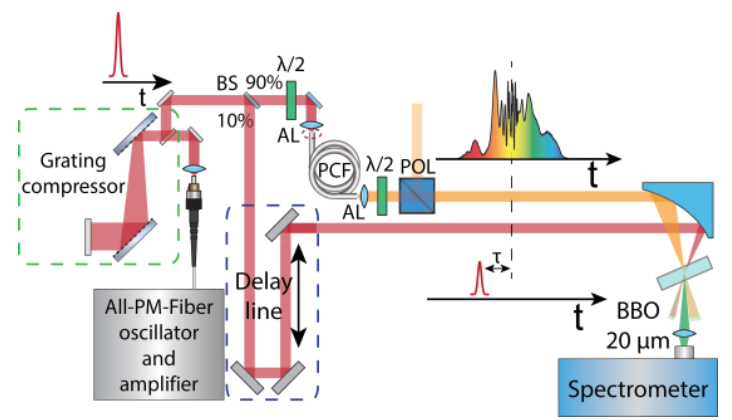

Fig. 3. The experimental XFROG scheme. BS - beam Splitter, $\lambda / 2-$ half-wave plate, AL - Aspheric Lens, PCF - Photonic Crystal Fiber, $\mathrm{POL}$ - polarizer, $\mathrm{BBO}-20 \mu \mathrm{m}$ thick barium borate crystal.

Increasing the pump power results in the broader spectrum of a supercontinuum pulse. From the NL-1050NEG-1 we achieved smooth Group Delay distribution without any discontinuities. The benefit of the broadband all-normal dispersion supercontinuum is the possibility to compress the pulse to very short durations [18], which we also presented in [19].

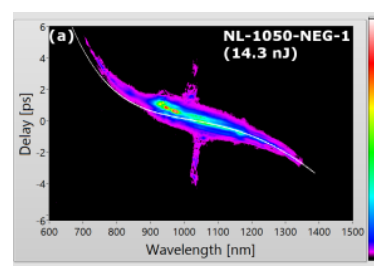

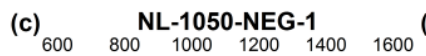

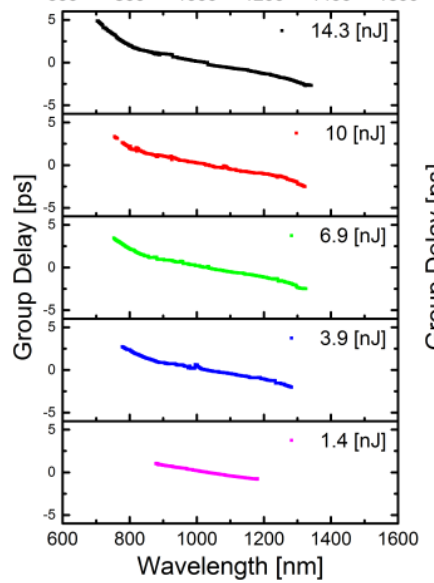

Fig. 4. The XFROGs' maps (a, b) show spectral intensities of sum frequency generation in respect to the delay of an ultrashort gate pulse. The white line on the map represents the theoretical GD calculated from the fiber dispersion data. The graphs (c, d) present pulses GD distributions for different energies of the pulse.

Crossing the ZDW during the broadening of the spectrum in the case of LMA-PM-5 fiber dramatically changes the characteristic of the output supercontinuum pulse. When 
the spectrum of the pulse during the broadening exceeds the ZDW, different frequencies contained in the broadened pulse spectrum overlap temporarily. Thus the spectrum is affected because of mutual interaction between these frequencies. Processes as cross-phase modulation (XPM) [20], resonant radiation [13], Raman solitons [21] and dispersive waves [12] can be distinguished in the XFROG maps measured for LMAPM-5 fiber.
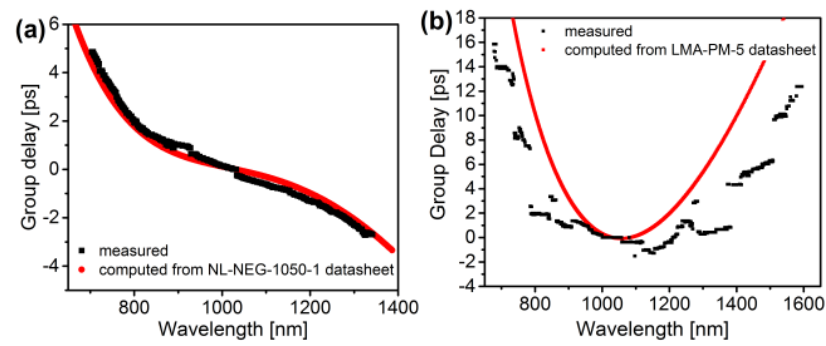

Fig. 5. Graphs show the differences between measured GD for NLNEG-1050-1 (a) and LMA-PM-5 (b) fiber in comparison with GD computed from datasheets of the fibers.

The last but not least result is the comparison between the measured data and that from the data presented in fibers datasheets (Fig. 5). For the NL-NEG-1050-1 fiber the standard deviation between the measured GD and computed is equal to 0.29 , which is a satisfying result. The differences between the measured and calculated GD from LMA-PM-5 dispersion profile are higher with a standard deviation of 5.26, which is caused by the strong influence of nonlinear processes which disturb the spectral broadening lead by the dispersion profile. Another important issue is the polarization state of different spectral components contained in the broad supercontinuum spectrum. Such changes in the polarization state are the source of hidden intrinsic noise [22]. The differences in the Polarization Extinction Ratio (PER) between orthogonal polarizations for PM (LMA-PM-5) and non-PM (NL-NEG-1050-1) fibers during pulse energy changes are presented in Fig. 6.

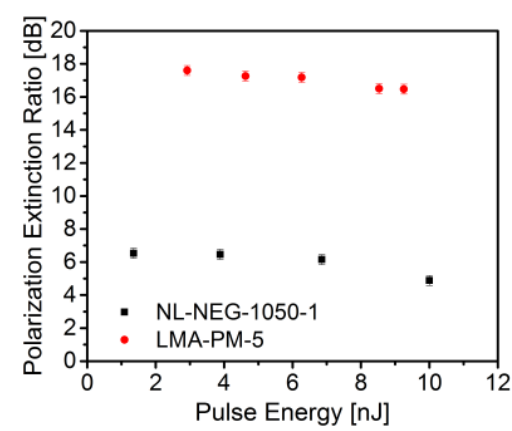

Fig. 6. Polarization Extinction Ratios measured for non-PM NL-NEG1050-1 fiber and LMA-PM-5 fiber.
The PER value for an LMA-PM-5 fiber is close to the fiber polarization extinction ratio specified by the producer $(>18 \mathrm{~dB})$ for all used energies. PER is decreasing with a growth of pulse energy which is caused by the nonlinear birefringence during the propagation of the pulse inside the fiber core. Using PM fibers allows maintaining the polarization state of a coupled seeding pulse.

Concluding, we have measured, with the XFROG method, dispersion maps for two kinds of fiber: the normal dispersion fiber and the fiber in which a broadened spectrum of the pulse crossed the ZDW. For the second fiber, the influence of several nonlinear processes, except the spectral broadening due to the SPM, was observed.

\section{References}

[1] M. Bradler, P. Baum, E. Riedle, Appl. Phys. B 97, 561 (2009).

[2] T. M. Kardaś, B. Ratajska-Gadomska, W. Gadomski, A. Lapini, R. Righini, Opt. Expr. 21, 24201 (2013).

[3] A. Brodeur, S.L. Chin, Phys. Rev. Lett. 80, 4406 (1998).

[4] R.R. Alfano, ed., The Supercontinuum Laser Source: Fundamentals with Updated References (Springer, 2006).

[5] A.L. Gaeta, Phys. Rev. Lett. 84, 3582 (2000).

[6] J.M. Dudley, G. Genty, S. Coen, Rev. Mod. Phys. 78, 1135 (2006).

[7] M. Klimczak, B. Siwicki, P. Skibiński, D. Pysz, R. Stępień, A. Heidt, C. Radzewicz, R. Buczyński, Opt. Expr. 22, 18824 (2014).

[8] D.J. Kane, R. Trebino, IEEE J. Quantum Electron. 29, 571 (1993).

[9] J. Dudley, X. Gu, L. Xu, M. Kimmel, E. Zeek, P. O’Shea, R. Trebino, S. Coen, R. Windeler, Opt. Expr. 10, 1215 (2002).

[10] N. Nishizawa, T. Goto, Opt. Expr. 8, 328 (2001).

[11] X. Gu, L. Xu, M. Kimmel, E. Zeek, P. O'Shea, A.P. Shreenath, R. Trebino, R.S. Windeler, Opt. Lett. 27, 1174 (2002).

[12] S. Roy, S.K. Bhadra, G.P. Agrawal, Opt. Lett. 34, 2072 (2009).

[13] S. Bose, S. Roy, R. Chattopadhyay, M. Pal, S.K. Bhadra, J. Opt. 17, 105506 (2015).

[14] T. Roger, M.F. Saleh, S. Roy, F. Biancalana, C. Li, D. Faccio, Phys. Rev. A 88, (2013).

[15] G.P. Agrawal, Nonlinear Fiber Optics (Elsevier/Academic Press, 2013).

[16] J. Szczepanek, T. Kardaś, M. Nejbauer, C. Radzewicz, Y. Stepanenko, Proc. SPIE 9728, 972827 (2016).

[17] C. Iaconis, I.A. Walmsley, IEEE J. Quantum Electron. 35, 501 (1999).

[18] L.E. Hooper, P.J. Mosley, A.C. Muir, W.J. Wadsworth, J.C. Knight, Opt. Expr. 19, 4902 (2011).

[19] J. Szczepanek, T.M. Kardas, Y. Stepanenko, Optical Society of America Frontiers in Optics Conference, FTu3C-2 (2016).

[20] G. Genty, M. Lehtonen, H. Ludvigsen, Opt. Expr. 12, 4614 (2004).

[21] S. Roy, S.K. Bhadra, K. Saitoh, M. Koshiba, G.P. Agrawal, Opt. Expr. 19, 10443 (2011).

[22] Y. Liu, Y. Zhao, J. Lyngso, S. You, W.L. Wilson, H. Tu, S.A. Boppart, J. Light. Technol. 33, 1814 (2015). 